\title{
Investigating familiar interactions to help older adults learn computer applications more easily
}

\author{
Nic Hollinworth and Faustina Hwang \\ School of Systems Engineering \\ University of Reading \\ Whiteknights \\ Reading, UK \\ RG6 6AY \\ \{n.d.p.hollinworth, f.hwang\}@reading.ac.uk
}

\begin{abstract}
Many older adults wish to gain competence in using a computer, but many application interfaces are perceived as complex and difficult to use, deterring potential users from investing the time to learn them. Hence, this study looks at the potential of 'familiar' interface design which builds upon users' knowledge of real world interactions, and applies existing skills to a new domain. Tools are provided in the form of familiar visual objects, and manipulated like real-world counterparts, rather than with buttons, icons and menus found in classic WIMP interfaces.
\end{abstract}

\begin{abstract}
This paper describes the formative evaluation of computer interactions that are based upon familiar real world tasks, which support multitouch interaction, involves few buttons and icons, no menus, no right-clicks or double-clicks and no dialogs. Using an example of an email client to test the principles of using "familiarity", the initial feedback was very encouraging, with 3 of the 4 participants being able to undertake some of the basic email tasks with no prior training and little or no help. The feedback has informed a number of refinements of the design principles, such as providing clearer affordance for visual objects. A full study is currently underway.
\end{abstract}

Human Computer Interaction, Older Adults, Multitouch Interaction, Familiarity, Email, non-WIMP interfaces

\section{INTRODUCTION}

With the proportion of older people in the UK population continuing to increase (Nat Stats, 2008), there is a need for older people to be able to take advantage of current technologies for work, recreation and communication with friends and family (Czaja and Lee, 2008). Many older adults wish to learn how to use computer applications, but find many of them difficult to use and are discouraged by counter-intuitive interfaces that have not been designed with consideration for the needs of older adult users (Arnott et al., 2004; Dickinson et al., 2005; Sayago and Blat, 2010). Hence, potential users may feel that learning to use a computer application is not worth the investment in time (Leonardi et al., 2010).

The current cohort of older adults have had relatively little exposure to computers compared with the younger generations (Arnott et al., 2004; Sayago and Blat, 2010), and since hardware and software was not designed for their particular set of cultural experiences, computer technologies can appear unfamiliar and alien (Leonardi et al., 2008). However, interaction styles are now drawing to a greater extent than ever before on users' everyday experience, using knowledge and understanding from a non-computer domain (Jakob et al., 2007). This presents new possibilities for performing computer tasks, and has the advantage of helping to break down cultural boundaries. Hence, our current work looks at the development of an interface that draws on users' existing experiences with real world objects, using their familiarity with these artefacts as a basis for learning how to use a computer application.

To demonstrate the principles of drawing on familiarity, a novel email client (which we have called tmail) has been developed for older adults that is not based on traditional input methods or standard WIMP interactivity involving icons, menus and buttons, but which is grounded in real life experiences of physical objects, and cast within the domain of writing and handling email messages and attachments. 


\section{RELATED WORK}

\subsection{Email applications for older adults}

Recent studies (Arnott et al., 2004; Czaja and Lee 2007; Hawthorn, 2003; Leonardi et al., 2010; Sayago and Blat, 2010) have shown that older adults find computer applications, such as email clients, difficult to learn and have difficulties remembering how to use them. Many applications suffer from an overload of features, most of which will not be used by older adult users. For example, in a recent study of older adults' interaction with the web, Sayago and Blat (2010) found that email was used quite infrequently - most users sent just 4-6 emails per month to their children and grandchildren, and about 3-5 per week to friends. Some 'common' functions such as saving an attachment or CC and BCC were not used, and participants just wanted a simple method of sending and receiving emails.

Alternative email applications for older adults have been studied widely. For example, Hawthorn (2003) developed SeniorMail, a redevelopment of Outlook aimed at simplifying some of the basic functionality of an email client, although the interface in this instance is still quite complicated. Arnott et al. (2004) started with the essential functionality that older users actually wanted to use in an email client, and created Simple Mail. The aim was to make tasks simpler, and create a system that is visually uncluttered and perceived as being easy to use. The interface, like SeniorMail was based upon standard GUI components, but kept deliberately simple. Dickinson et al. (2005) also developed an email application (Cybrarian), aimed at novice older adult computer users, which implemented only the essential functionality for a working system. They kept the number of buttons or actions to less than 10 per screen, avoided scrollbars (which older adults find difficult to operate) and used terminology understandable to a person of this age group.

Whilst offering a simpler interface and limiting unnecessary functionality, all of these examples still rely on knowledge of classic WIMP interfaces. Anyone unfamiliar with this style of interaction would need to invest a great deal of time and effort in learning how to use both the hardware and software, since there is little, if anything, in these standard interfaces with which to orient users.

\subsection{Familiar interfaces for older adults}

One possible approach for improving the learning and understanding of computer applications for older adults is to design the interface around objects that are familiar to the user, through physical features and behaviours they are already acquainted with in the real world (see Leonardi et al., 2008, 2010). Hence, rather than being forced to adapt to new paradigms or learn a new (technological) language, users can apply previous experience, perhaps from a non-computing domain, to accomplish basic tasks on the computer (Leonardi et al, 2008). A great advantage of this approach is that basic knowledge of the real world, perhaps learned since childhood, is more highly practiced and so should take less mental effort (Jakob et al., 2007).

Leonardi et al. (2010) looked at the idea of familiarity in designing an interface for older adults, and created a touch-based interface for older adults that used a combination of gestures and drag and drop to facilitate actions. In their example, they used objects resembling postcards for sending messages and a chest of drawers graphic to represent a facility for storing images and messages. Messages were 'posted' by dragging a message symbol onto a receiver's mailbox icon.

As with other systems that have been developed for older adults, we aim to simplify the functionality as a means of simplifying the interface. In contrast, though, we do not use standard WIMP interaction, opting instead for an interface that employs familiar visual objects, with familiar behaviours. However, unlike Leonardis' et al. (2010) approach (using standard drag and drop) objects should be used and behave predictably, like similar objects would in the real world. Complex tasks, such as adding an attachment to an email, are reduced to simple manipulations, such as sliding an image over the desktop and onto a message to attach it.

Our overall idea is to encourage users to learn how to accomplish basic tasks by using their existing knowledge of familiar everyday objects, without having to rely on learning complicated sequences of actions, which can deter novices. We believe this approach can help older adult computer users attain a greater sense of ownership in their learning, a greater degree of autonomy and have to rely less on external help.

\section{DESIGN PRINCIPLES}

The aim of this research is to investigate the potential for "familiar" interaction to improve computer access for older adults. The idea is to build upon users' knowledge of real world interactions and apply their existing skills to a new domain (i.e. computing), in order to improve learnability and discoverability, and help reduce anxiety. 
Rather than present users with buttons, icons and menus found in classic WIMP interfaces, we wanted to provide tools in the form of familiar visual objects that can be manipulated like real-world counterparts in order to accomplish basic tasks within an application. As existing experience and familiarity with objects would be used, this could help to reduce cognitive load (Jakob et al., 2007; Norman, 2011), and so be of benefit to all users, particularly older adults who often find the sequences of tasks in conventional application overly complex and difficult to recall (Czaja and Lee, 2008; Hawthorn, 2000). For example, given an object resembling a piece of paper, in the real world most people would know through experience that you can place a finger on it and slide it across the surface on which it rests. Therefore, providing a similar object within an application interface would give users a head start in terms of recognising its potential use, and how to make use of it.

\section{IMPLEMENTATION OF DESIGN}

The tmail application uses a desktop metaphor, with 'unnecessary' functionality (i.e. rarely used features) omitted in order to maintain simplicity and keep perceived complexity to a minimum. See figure 1.

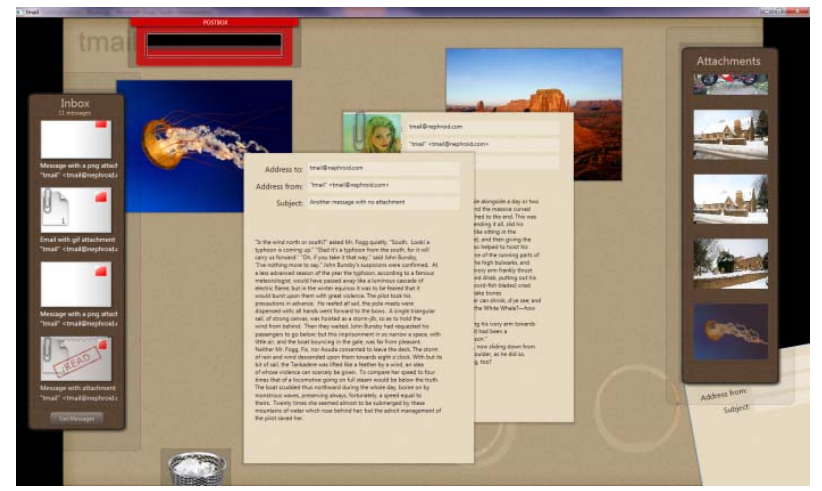

Figure 1: tmail employs visual objects that are familiar to users, and behave predictably.

\subsection{Familiar visual objects}

Visual objects are modelled on paper-based letters and photographs (for attachments) with the design of objects being familiar to the user through their similarity to the real world (Leonardi et al., 2008). For example, a message resembles a typed letter on paper, with attachments displayed as small images fastened to the message with a paperclip. See Figure 2.

Objects used in the tmail interface are designed to be familiar to users, and resemble objects that in the real world would be used to perform similar tasks. For instance, email messages shown in the inbox resemble paper envelopes. When a message is dragged onto the desktop for reading, the envelope icon representing that message changes to one which is torn open to show that it has been read. Messages are sent by sliding them into a postbox, and messages are deleted by dragging a message into a waste paper basket.

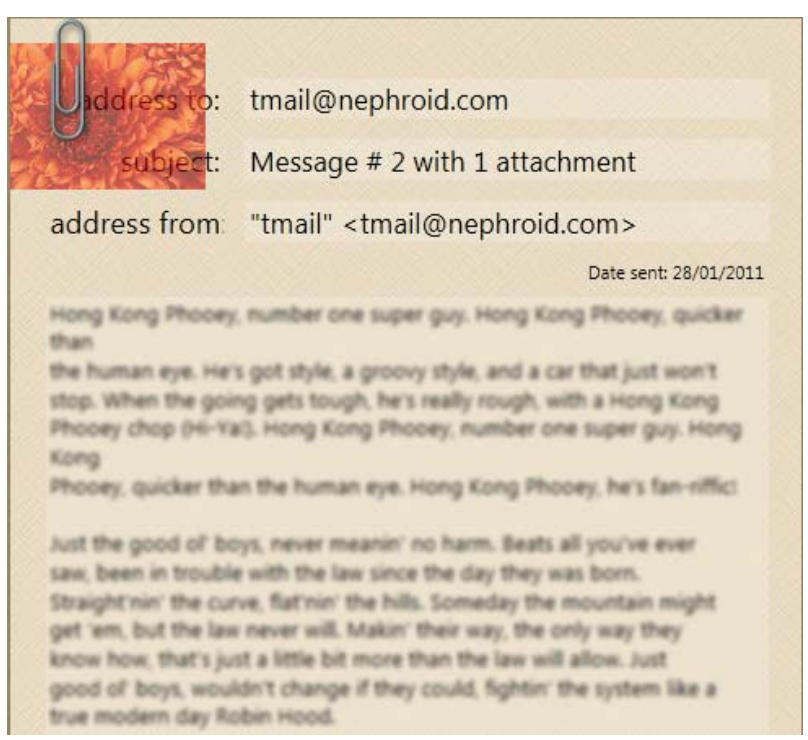

Figure 2: An email message (reduced in height to fit) with an attachment shown at the top left of the message.

To make objects easier to select, they need to be large, particularly as older users may have reduced manual dexterity (Czaja and Lee, 2008). Hence, visual objects were designed to be deliberately large, and with large contact areas for selecting with touch. For example, the size of the envelope icons used in the inbox is 155 pixels $x 100$ pixels much larger than a standard desktop icon. This helps to make them easier to handle when dragging to the desktop, and the large appearance also makes it easier to see the state of the message - whether it was read, unread, has an attachment or is a draft.

\subsection{Familiar behaviours with objects}

Most tasks are performed with the tmail interface by touching and moving objects, similar to the way in which objects are manipulated in the real world. For instance, a message or image can be moved across the desktop by touching and sliding, and an attachment can be viewed by 'unclipping' it from the message by tugging it out of the paperclip. One of the advantages of the multitouch screen is that objects, such as images, can be simultaneously rotated and translated whilst they are being dragged - a behaviour that most people will already be familiar with when handling real objects.

Arguably, touchscreen interaction also has its pitfalls, particularly with actions such as the standard drag and drop. This can be very 
demanding for older computer users, who may be prone to dropping the object prematurely (Leonardi et al., 2010). To overcome such problems, we use a modified form of drag and drop for most (but not all) operations, in which objects stay where you drop them, and do not return to the starting location. This behaviour might also better fit the user's expectations, and may add to the realism.

\subsection{Removing seldom used functionality}

To keep the interface simple and keep perceived complexity to a minimum [see Dickinson, Arnott, Hawthorn], we omitted some functionality that is seldom used by older adults. For example, there is no provision in the application for email options such as CC or BCC, since these are facilities that are used very infrequently by older adult computer users (Sayago and Blat, 2010). In addition, the facility to organise messages into different folders was also omitted, as this was considered to be an additional complication for users, and one that would probably not be used and would not be missed (Sayago and Blat, 2010).

\subsection{Avoiding hidden functions}

Tasks in a standard Windows environment often involve a complex sequence of actions, even if they appear relatively simple. For example, adding an attachment to an email message often involves quite a large number of steps, including locating the menu option (or button) for adding the attachment, browsing the file system for the necessary file, etc. This can cause difficulties for older adults as cognitive changes and short-term memory decline can make it more difficult to learn and recall complex sequences (Arnott et al, 2004; Czaja and Lee, 2008). Hence, we wanted to keep tasks as simple as possible, with few or no hidden conditions that need to be remembered, and with an emphasis on keeping the number of task steps to a minimum. Thus the available functionality was designed to be as explicit as possible and 'visible' to the user (Norman, 2010) in the sense that users should be able to easily discover for themselves what the application is capable of, and require little or no help in understanding what the interface offers.

As an example, unlike most classic windows applications, we did not employ dialogs in tmail, and so adding an attachment only requires the user to drag the file from the panel or desktop directly onto the message in order to attach it. As no dialogs are used, file attachments are placed in a single location, which has the advantage that users do not have to search in other folders or disk drives looking for attachments they have saved.
Similarly, when replying or forwarding a message, no additional actions are required by the user, such as selecting a button for reply or forward. Unlike conventional email applications, only the recipient address needs to be modified to perform this task.

\subsection{Some non "real-world" aspects}

Whilst much of the functionality and visual appearance of the interface is based upon familiar real world objects and actions, there are some instances where this could not be easily implemented, or does not have a real-world counterpart. For instance, using a two finger spread or pinch (see Saffer, 2009), messages and attachments can be scaled to make them easier to view, but there is nothing similar to this action that can be performed in the real world. Whilst this is an action that is not immediately apparent from the appearance of the object, it can be learned quite easily (see section 6 below), and is a skill that transfers to many other devices too.

Similarly, the attachment panel and inbox use a scrolling list to enable the display of more items using a flick gesture, but there is nothing similar in the real world. Perhaps a more realistic technique would be to use a stack of messages, rather than a list, but we chose this mechanism as it is quite an efficient way of displaying several messages (or images) at a time. In addition, messages still rely upon keyboard input, although we have tried to make this as 'real' as possible by allowing typing anywhere within the message body, without being constrained to lines and line breaks.

\section{FORMATIVE STUDY}

The tmail application was evaluated in a formative study to gain some initial feedback from users and see how well the design supported learnability and discoverability. Three older adult volunteers (aged 53, 54 and 67) and one younger volunteer (aged 30) participated in the study. Each volunteer had at least 3 years computer experience using a standard email client, and also experience of web browsing and general applications such as word processors and spreadsheets. None of the participants had any diagnosed visual impairments or motor impairments that would give rise to severe difficulties with using a touchscreen. Participants had normal or corrected to normal eyesight.

\subsection{Apparatus}

The pilot study was conducted using a Dell Latitude E5500 laptop, running Windows 7 Professional, and connected to a 19inch $3 \mathrm{M}$ multitouch monitor at a resolution of $1280 \times 800$. The touchscreen was 
mounted vertically. Both a physical keyboard and an onscreen keyboard facility (provided by Windows 7) were used in the study.

\subsection{Method}

Each participant was given a set of 12 basic email tasks to complete using tmail, such as finding a specific message in the inbox, displaying a message for reading, sending a message to a specific address and adding and viewing an attachment. Participants were given no training on how to use the application, and were only given help if they requested it.

Qualitative data on the user's experience was captured by one of the researchers through observation and an interview of approximately 20 minutes. During the trials, participants were asked to 'think aloud' so that we could capture their thoughts and perceptions of the application as they discovered how to use it. Data was captured on the problems that were experienced when attempting the tasks, which ones were found to be particularly easy or straightforward, suggestions for improvements and when help or clarification was requested. Participants were also asked what they liked and disliked most about the application, and what functions of an email program they would probably use themselves.

\section{RESULTS}

Participants generally found the visual objects in the interface easy to understand, and all four managed to work out how to view messages (by dragging an object from the inbox onto the desktop) after a little trial and error. Two of the older participants and the younger participant initially tried to 'click' on an unread message to view it, and when this did not immediately occur, they tried dragging the message icon vertically. This just scrolled the list, but after a few more attempts they each worked out that the icon needed to be dragged horizontally. This 'accidental' scrolling had the advantage of showing them how to view more messages.

One (older) participant figured out how to read messages and view attachments within the first minute, although they did not immediately understand that the inbox could be scrolled to display further messages. This latter point could be due to the omission of a suitable visual cue, and is an issue being considered further.

All four participants used a single finger to select objects to begin with, and were unaware that the touch screen supported actions using more than one finger. Once a brief demonstration of scaling and rotating an image was given, two participants (one young, one older) began experimenting with the objects on the desktop, and attempted rotations, scaling and translations with some of the other visible objects. The other two participants were a little reluctant to experiment, preferring to be given instructions on how the interface was used. A popular feature with all participants was the ability to scale a message with a two finger spread.

All participants generally agreed that they liked the mechanism for adding and viewing attachments by dragging and dropping, as it required a single action, and there was no need to go through a dialog to locate the attachment when adding. One older participant commented that they liked the attachment previews (as thumbnail images), as it helped in determining whether they wished to view the attachment.

All four participants requested some method of 'tidying' the desktop, as it could become cluttered quite easily with messages and attachments due to their size. None of the participants attempted to 'stack' images or messages to clear space on the desktop (which is often found when dealing with paper documents and folders (see Barreau and Nardi, 1995)), but instead moved the messages out of the immediate area in which they were working.

\section{CONCLUSIONS}

The use of familiarity in an interface is not a new idea, having been exploited by game designers for almost two decades. However, the use of familiarity to this extent within everyday desktop applications is still relatively underexplored and has much potential to improve computer access for older adults.

This formative study suggests that familiar interfaces can help in orienting older users with an application, and that functionality can be made more explicit by exploiting prior knowledge and skills of the real world that people already possess. Actions in many cases can be made much simpler and are easily reversible, which can help to encourage novice users to explore what an application has to offer. More importantly, users are not forced to learn a new technological language or rote memorise complex task sequences which have no grounding within their own culture or prior experiences.

\section{FUTURE WORK}

\subsection{Implementation of functionality}

The current version of tmail has several limitations which will be addressed before the full study takes place, and we are aiming to include further 
functionality. In particular, html emails are not yet supported, so images cannot yet be embedded inline into the email body and only image attachments (png, gif and jpeg) are currently supported. Although it is possible to attach other file types to a tmail message, only image types can be viewed within the application.

\subsection{Further Enhancements}

A future version of tmail will include the ability to write emails by hand, using a stylus in one hand and positioning the email message using the other, rather like the way in which most people write a letter on paper. A prototype for this functionality has already been constructed, but the quality of writing is still quite poor due to the lack of a suitable stylus that will work effectively with capacitive touchscreens. The newer resistive touchscreens that support multiple contact points may be able to offer this functionality in the near future.

\subsection{Full Study}

A full study is currently being planned and is to be conducted using a group of older adult computer users of varied experience, with a comparison being made between the tmail application described in this study and a regular web-based email client (Gmail). The same set of (12) tasks from this formative study will be used, but undertaken on two separate occasions separated by a two week interval. Data will be collected on the amount of help requested, the number of errors made in performing a task, and the number of verbal confirmations given. Using this data we can obtain a measure of discoverability by considering the amount of help required by a participant - the more times help is requested for a specific task, the less discoverable it is considered to be. Learnability can be measured with this set of data, too, by considering whether the help, errors and confirmations decreased after the first session, or not.

\section{ACKNOWLEDGMENTS}

Thanks to the staff and volunteers at Age UK Reading, Age UK Banbury, 3M, and the University of Reading. This work is funded by School of Systems Engineering.

\section{REFERENCES}

Arnott, J. L., Khairulla, Z., Dickinson, A., Syme, A., Alm, N., Eisma, R., and Gregor, P. (2004) Email interfaces for older people, Proc of the IEEE
International Conference on Systems, Man and Cybernetics, Netherlands, 111-117.

Barreau, D. and Nardi, B. A. (1995) Finding and reminding: file organization from the desktop. SIGCHI Bull, 27, 3, 39-43.

Czaja, S. J. and C. C. Lee. (2007) The impact of aging on access to technology, Universal Access in the Information Society, 5, 4, 341-349.

Czaja, S. and Lee, C.C. (2008) Information Technology and Older Adults, The Human Computer Interaction Handbook, Sears, A and Jacko, J. (eds), CRC Press, 777-792.

Dickinson, A., Newell, A.F., Smith, M.J. and Hill, R.L. (2005) Introducing the Internet to the over-60s: developing an email system for older novice computer users, Interacting with Computers, 17, 122.

Hawthorn, D. (2000) Possible implications of aging for interface designers, Interacting with computers, $12,507-528$.

Hawthorn, D. (2003) How universal is good design for older users?, Proceedings of the 2003 Conference on Universal Usability, Vancouver, Canada, 38-45.

Jakob, R., Girouard, A., Hirshfield, L., Horn, M., Shaer, O., Solovey, E. and Zigelbaum, J. (2007) Reality-Based Interaction: Unifying the New Generation of Interaction Styles, Proc CHI 2007, San Jose, California, 2465-2470.

Leonardi, C., Mennecozzi, C., Not, E., Pianesi, F. and Zancanaro, M. (2008) Designing a Familiar Technology for Elderly People, Proc of the 6th International Conference of the International Society for Gerontechnology, ISG'08, Pisa, Italy.

Leonardi, C., Albertini, A., Pianesi, F. and Zancanaro, M. (2010) An Exploratory Study of a Touch-based Gestural Interface for Elderly, NordCHI' 10, Reykjavik, Iceland, 845-850.

Norman, D. (2010) Natural User Interfaces are Not Natural, ACM Interactions, May/June.

Norman, D (2011) Living with Complexity, MIT Press.

Saffer, D. (2009) Designing Gestural Interfaces, OReilly, Cambridge.

Sayago, S. and Blat, J. (2010) Telling the story of older people emailing: an ethnographical study. Int J Human-Computer Studies, 68(1), Duluth: Academic Press, 105-112.

UK National Statistics.

http://www.statistics.gov.uk/cci/nugget.asp?ID=949, Last viewed on 12th January 2010 